Bull. Austral. Math. Soc.

$06 \mathrm{~F} 20,46 \mathrm{~A} 04,46 \mathrm{~A} 40$

VOL. 58 (1998) [383-386]

\title{
FRÉCHET AL-SPACES HAVE THE DUNFORD-PETTIS PROPERTY
}

\author{
J.C. Díaz, A. Fernández and F. Naranjo
}

A Fréchet lattice $E$ is an AL-space if its topology can be defined by a family of lattice seminorms that are additive in the positive cone of $E$. Grothendieck proved that AL-Banach spaces have the Dunford-Pettis property. This result was recently extended by Fernández and Naranjo to AL-Fréchet spaces with a continuous norm and weak order unit. In this note we show how to remove both hypotheses.

Dunford and Pettis proved in [4] that every weakly compact operator from $L_{1}(\mu)$ into itself carries weakly convergent sequences onto norm convergent sequences.

In general it is said that a Hausdorff locally convex space $E$ has the DunfordPettis property (following [7, Definition 1]) if for any Banach space $F$ and every linear continuous mapping $T: E \rightarrow F$ that carries bounded sets of $E$ to weakly relatively compact sets of $F$, then $T(C)$ is relatively compact in $F$ for any absoutely convex, weakly compact subset $C$ in $E$.

This property has been intensively studied and characterised in different contexts. In particular, Grothendieck [7] established that both classes of Banach AL and AM spaces possess the Dunford-Pettis property. (See also [1, Theorem 19.6].)

This result was proved by using the duality between these classes, together with the representation of AL- and AM-spaces by means of spaces of integrable functions and continuous functions, respectively. First of all Grothendieck proved that AM-spaces have the Dunford-Pettis property. For AL-spaces it then follows by duality.

The concept of generalised AL-spaces was introduced by Wong in [9] (see also [10]) in the setting of locally convex lattices.

We recall that a Fréchet lattice $E$ is said to be a Fréchet AL-space if its topology can be defined by a family of lattice seminorms $|\cdot|$ that are additive in the positive cone $E^{+}$of $E$, that is,

$$
|x+y|=|x|+|y|, \quad x, y \in E^{+} .
$$

In [5] the second and third named authors proved, by using a representation result for certain Fréchet lattices due to Dodds, de Pagter and Ricker, [3], that every Fréchet

Received 12th February, 1998

The research has been partially supported by La Consejería de Educación y Ciencia de la Junta de Andalucía and DGICYT projects number PB94-1460 and PB94-0441.

Copyright Clearance Centre, Inc. Serial-fee code: 0004-9729/98 \$A2.00+0.00. 
AL-space with a continuous norm and weak order unit has the Dunford-Pettis property. Here we establish that every Fréchet AL-space has the Dunford-Pettis property. Let us observe at this point that there is no well established duality relation between locally convex AL-spaces and AM-spaces.

The proof will require some preliminary results.

LEMMA 1. Every separable closed vector subspace of a Fréchet lattice is contained in a closed separable Fréchet sublattice.

Proof: See [1, Exercises 8 and 9 (p.197)].

Lemma 2. If ali closed and separable sublattices of a Fréchet lattice $E$ have the Dunford-Pettis property, then $E$ also has this property.

Proof: Let $F$ be a Banach space and let $T: E \rightarrow F$ be a linear continuous mapping for which $T(B)$ is weakly relatively compact in $F$ for any bounded set $B$ in $E$. Taking into account $\left[8\right.$, Theorem 1] it suffices to prove that $\left(T\left(x_{n}\right)\right)_{n}$ is a Cauchy sequence in $F$, for any weakly Cauchy sequence $\left(x_{n}\right)_{n}$ in $E$.

Consider the closed and separable sublattice $H$ which is generated by the sequence $\left(x_{n}\right)_{n}$. The restriction mapping $T: H \rightarrow F$ is linear, continuous and carries bounded sets of $H$ into weakly relatively compact sets of $F$. Since $H$ has the Dunford-Pettis property and $\left(x_{n}\right)_{n}$ is weakly Cauchy in $H$, it follows that $\left(T\left(x_{n}\right)_{n}\right)$ is a Cauchy sequence in $F$.

Proposition 3. Let $E$ be a Fréchet $A L$-space with a continuous norm. Then $E$ has the Dunford-Pettis property.

Proof: Let $H$ be any closed and separable sublattice of $E$. Then $H$ is a Fréchet AL-space with a continuous norm and a weak order unit. From [5, Corollary 4.5] $H$ has the Dunford-Pettis property. Now apply Lemma 2 to obtain the result.

PROPOSITION 4. Let $\left(E_{n}\right)_{n}$ be a sequence of Fréchet spaces, each one with the Dunford-Pettis property. Then, the product Fréchet space $E:=\prod_{n=1}^{\infty} E^{n}$ also has the Dunford-Pettis property.

Proof: Fix, in every $E_{k}$, a countable increasing system $\mathcal{P}_{k}$ of continuous seminorms giving its topology. Let $x=\left(x_{n}\right)_{n} \in E$, with $x_{n} \in E_{n}$. Then the topology of $E$ is determined by the family of seminorms $H_{k}$ given by

$$
\left|\left(x_{n}\right)_{n}\right|_{k}:=\sum_{j=1}^{k}\left|x_{j}\right|_{k}, \quad k=1,2, \ldots
$$

where $\left|x_{j}\right|_{k}$ is the value of the $k$-th seminorm of $\mathcal{P}_{j}$ acting on the element $x_{j} \in E_{j}$. 
Let $F$ be a Banach space with norm $\|\cdot\|$ and let $T: E \rightarrow F$ be a linear and continuous mapping such that $T(B)$ is weakly relatively compact in $F$ for every bounded $B$ in $E$. Since $T: E \rightarrow F$ is continuous, there are constants $M>0$ and $k \geqslant 1$ (that we fix) such that

$$
\|T x\| \leqslant M|x|_{k}, \quad x \in E .
$$

If $G:=\left\{\left(x_{n}\right)_{n} \in E: x_{n}=0\right.$, for all $\left.n>k\right\}$ then we have $|x|_{k}=0$ for all $x \in G$. From (0.1) we deduce that $T(x)=0$ for every $x \in G$. Therefore, $T$ admits a natural factorisation through the space $\prod_{j=1}^{k} E_{j}$. Now the result follows because the Dunford-Pettis property is inherited by finite products.

We are now ready to establish the main result.

THEOREM 5. Every Fréchet $A L$-space has the Dunford-Pettis property.

Proof: Let $E$ be a Fréchet AL-space. Then $E$ has the Lebesgue property [6, Theorem 2]. Now, from [2, Remark 2 of Theorem 1] we know that either $E$ has a continuous norm or $E$ is isomorphic to the product of a sequence of Fréchet spaces each one having a continuous norm. In the first case, $E$ has the Dunford-Pettis property by applying Proposition 3. In the second case, we obtain the result by applying Proposition 3 and Proposition 4.

\section{REFERENCES}

[1] C.D. Aliprantis and O. Burkinshaw, Positive operators, Pure and Applied Mathematics 119 (Academic Press, Orlando, Fl., 1985).

[2] J.C. Díaz, 'Continuous norms on Fréchet lattices', Arch. Math. (Basel) 52 (1989), 155-158.

[3] P.G. Dodds, B. de Pagter and W.J. Ricker, 'Reflexivity and order properties of scalar-type spectral operators in locally convex spaces', Trans. Amer. Math. Soc. 293 (1986), 355-380.

[4] N. Dunford and P.J. Pettis, 'Linear operations on summable functions', Trans. Amer. Math. Soc. 47 (1940), 323-392.

[5] A. Fernández and F. Naranjo, 'Operators and the space of integrable scalar functions with respect to a Fréchet-valued measure', J. Austral. Math. Soc. Ser. A 64 (1998), 1-18.

[6] K.G. Grosse-Erdman, 'Lebesgue's theorem of differentiation in Fréchet lattices', Proc. Amer. Math. Soc. 112 (1991), 371-379.

[7] A. Grothendieck, 'Sur les aplications linéaires faiblement compactes d'espaces du type $\mathcal{C}(K)$ ', Canad. J. Math. 5 (1953), 129-173.

[8] S.S. Khurana, 'Dunford-Pettis property', J. Math. Anal. Appl. 65 (1978), 361-364.

[9] Y.C. Wong, 'Characterizations of the topology of uniform convergence on order-intervals', Hokkaido Math. J. 5 (1976), 164-200. 
[10] Y.C. Wong and F.K. Ng, 'Nuclear and AL-spaces', Southeast Asian Bull. Math. J. 5 (1981), 45-58.

Dpto. Matemáticas

E.T.S.I.A.M.

Universidad de Córdoba

14004 - Córdoba

Spain

e-mail: ma1dialj@uco.es

Dpto. Matemática Aplicada II

Escuela Superior de Ingenieros

Camino de los Descubrimientos $\mathbf{s} / \mathbf{n}$

41092 - Sevilla

Spain
Dpto. Matemática Aplicada II

Escuela Superior de Ingenieros

Camino de los Descubrimientos s/n

41092 - Sevilla

Spain

e-mail: anfercar@matinc.us.es 